\title{
The Retrograde Spread of Synaptic Potentials and Recruitment of Presynaptic Inputs
}

\author{
Brian L. Antonsen, Jens Herberholz, and Donald H. Edwards \\ Brains and Behavior Program and Department of Biology, Georgia State University, Atlanta, Georgia 30302-4010
}

Lateral excitation is a mechanism for amplifying coordinated input to postsynaptic neurons that has been described recently in several species. Here, we describe how a postsynaptic neuron, the lateral giant (LG) escape command neuron, enhances lateral excitation among its presynaptic mechanosensory afferents in the crayfish tailfan. A lateral excitatory network exists among electrically coupled tailfan primary afferents, mediated through central electrical synapses. EPSPs elicited in LG dendrites as a result of mechanosensory stimulation spread antidromically back through electrical junctions to unstimulated afferents, summate with EPSPs elicited through direct afferentto-afferent connections, and contribute to recruitment of these afferents. Antidromic potentials are larger if the afferent is closer to the initial input on LG dendrites, which could create a spatial filtering mechanism within the network. This pathway also broadens the temporal window over which lateral excitation can occur, because of the delay required for EPSPs to spread through the large LG dendrites. The delay allows subthreshold inputs to the LG to have a priming effect on the lateral excitatory network and lowers the threshold of the network in response to a second, short-latency stimulus. Retrograde communication within neuronal pathways has been described in a number of vertebrate and invertebrate species. A mechanism of antidromic passage of depolarizing current from a neuron to its presynaptic afferents, similar to that described here in an invertebrate, is also present in a vertebrate (fish). This raises the possibility that short-term retrograde modulation of presynaptic elements through electrical junctions may be common.

Key words: sensorimotor; sensory neurons; crustacea; behavior; escape; gap junction

\section{Introduction}

The array of primary sensory afferents on a body surface conveys a continuously changing pattern of input to the CNS to represent the corresponding pattern of sensory stimuli that impinge on the animal. This pattern of input is not an absolutely faithful representation of the sensory stimuli: nonlinear sensory transduction, time-dependent and history-dependent receptor properties, and lateral inhibitory connections among afferents are widespread and act to distort the pattern of receptor responses so as to enhance signals of biological importance and to reduce other signals. Less common is the spread of excitation among afferents, leading some afferents to become excited in the absence of direct stimulation of the sensory receptors that normally drive them. One prominent example of this "lateral excitation" is found in the array of cones in the vertebrate retina, where gap junctions between cones enable the photoresponse of a cone to spread to its neighbors (Laughlin, 2002). Another recent example arises from the electrical coupling between primary mechanosensory afferents in the crayfish lateral giant (LG) tailflip escape circuit (Herberholz et al., 2002). These afferents are interconnected through a "lateral excitatory network" of electrical coupling, enabling un-

Received 0ct. 26, 2004; revised Feb. 10, 2005; accepted Feb. 11, 2005

This work was supported by National Institutes of Health Grant R01 NS26457 and National Science Foundation Grant 0349042.

Correspondence should be addressed to Dr. Brian L. Antonsen, Department of Biology, Georgia State University, P.0. Box 4010, Atlanta, GA 30302-4010. E-mail: biobla@langate.gsu.edu.

D01:10.1523/JNEUROSCI.4433-04.2005

Copyright $\odot 2005$ Society for Neuroscience $\quad$ 0270-6474/05/253086-09\$15.00/0 stimulated afferents within a sensory nerve to be recruited by simultaneously active neighboring afferents. Recruitment of unstimulated afferents requires a large initial stimulus, resulting in excitation from a critical density of coupled afferents. In both of these examples, some primary sensory neurons are excited not by direct sensory stimulation, but rather by the spread of neural excitation from neighboring stimulated sensory neurons.

Another possible mechanism of sensory neuron excitation is through antidromic spread of postsynaptic current from a postsynaptic neuron. Work in crayfish and goldfish has shown that gap junctions linking primary afferents to first-order interneurons can enable postsynaptic depolarizing currents to pass antidromically into presynaptic afferents and affect their excitability (Pereda et al., 1995; Herberholz et al., 2002; Curti and Pereda, 2004). This raises the interesting possibility that EPSPs in a postsynaptic cell can help recruit additional inputs to that cell by spreading antidromically into unexcited presynaptic elements. Such a positive feedback mechanism might be particularly useful in triggering high-threshold neurons such as those that mediate escape responses.

We have examined this possibility in the neural circuit for escape in crayfish, where an array of primary mechanosensory afferents converges on the dendrites of the LG. Here, we show that electrical synapses between primary afferents and the LG enable LG EPSPs to spread antidromically, affecting recruitment of additional afferent inputs. We ask how this capability contributes to the integrative properties of the LG neuron and its sensitivity to converging phasic inputs. We conclude that rather than 
providing a simple pathway for converging afferent input onto the LG, the electrical synapses mediate a temporally complex bidirectional pattern of transmission that spreads excitation among the afferent population as the LG is excited. The LG dendrites not only integrate the afferent EPSPs, they provide a current path between the converging afferent projections that enables excitation to spread across the afferent population.

\section{Materials and Methods}

Animals and experimental procedure. Crayfish (Procambarus clarkii) of both sexes and between 3 and $4 \mathrm{~cm}$ were obtained from commercial suppliers and kept in communal tanks for not more than 2 weeks before experiments. Animals were anesthetized on ice for 20-30 min. Dissections and experiments were performed in physiological saline of the following composition (in mM): $202 \mathrm{NaCl}, 5.37 \mathrm{KCl}, 13.53 \mathrm{CaCl}_{2}, 2.6$ $\mathrm{MgCl}_{2}$, and 2.4 HEPES, pH 7.4. The abdominal ventral nerve cord was exposed by removing the dorsal exoskeleton, viscera, and axial musculature, and the musculature of the tailfan was removed as completely as possible without straining or damaging the peripheral nerves of the sixth ganglion (A6) and while cutting or damaging as few primary afferent neurons as possible. Because of the necessity to remove the dorsal exoskeleton from abdominal segment 6 and part of the dorsal anterior telson to allow access for the electrodes, some or all of the branches of nerves 1 (N1) and 4 of A6, the nerves innervating these areas, were cut as far from the ganglion as possible. N2 and N3 of ganglia A1-A5 were cut, again because their targets were removed during dissection, whereas N1 (swimmerets) was left intact. The ventral exoskeleton, head, and most of the tailfan were left intact. For dye-filling experiments, the dorsal sheath was removed from A6 and a short length of the adjacent anterior connective, and A6 was gently supported on a small piece of Sylgard (Dow Corning, Midland, MI); for strictly physiological experiments, the cord was not desheathed. For all experiments involving cell filling or dye coupling, the preparation was bathed in circulating physiological saline maintained at $12^{\circ} \mathrm{C}$; all other experiments were done at room temperature without circulation.

Dye coupling. The LG on one side of the nerve cord was filled with Neurobiotin (NB) (Vector Laboratories, Burlingame, CA) and with 10,000 molecular weight (MW) dextran-linked Texas Red (TR) (Molecular Probes, Eugene, OR). This combination of tracers allows clear separation of the injected cell that contains both tracers, and the cells that dye couple to it, which will contain only NB that has passed through gap junctions (Peinado et al., 1993; Hatton and Yang, 1994; Johnson et al., 2000). This was done by penetrating the LG axon close to A6 with a low-resistance (5-10 M $\Omega$ ) electrode filled with a mixture of $2 \% \mathrm{w} / \mathrm{v} \mathrm{NB}$ and $0.7 \% \mathrm{w} / \mathrm{v}$ TR in $0.15 \mathrm{M} \mathrm{KCl}$ (Fig. 1). This solution was picospritzed using a PV820 pneumatic picopump (WPI, Sarasota, FL) very slowly (total time up to $2 \mathrm{~h}$ in some cases) into the LG axon. The end point of the injection was determined visually, when the TR could be clearly seen under transmitted light entering the larger dendritic branches of the LG in A6. At this point, the injection electrode was removed, and an additional 20 min were given to ensure complete spread of the tracers to the fine $\mathrm{LG}$ dendrites.

To determine the effect of peripheral nerve stimulation on dye coupling between the LG and primary afferents, we used glass pins to restrain $\mathrm{N} 1$ and $\mathrm{N} 3$ of A6 and to isolate N2 for stimulation with a suction electrode placed $1 \mathrm{~mm}$ from the ganglion (Fig. 1). A recording electrode (30 $\mathrm{M} \Omega, 3 \mathrm{M} \mathrm{KCl}$ ) was placed in the axon of the LG ipsilateral to the suction electrode, and the threshold voltage for firing the LG was determined by slowly raising the voltage of $0.1 \mathrm{~ms}$ pulses delivered to N2 from a Grass S88 stimulator (Grass Telefactor, West Warwick, RI). In some experiments, recording electrodes $(60-70 \mathrm{M} \Omega, 3 \mathrm{M} \mathrm{KCl})$ were also placed in the dendrites of the ipsilateral LG to record responses closer to the site of the afferent contacts. N2 was stimulated for $10 \mathrm{~min}$ at $70 \%$ of the LG threshold voltage. Six preparations were used at each of six stimulus regimens: unstimulated and 5, 10, 20, 50, and $100 \mathrm{~Hz}$. After a $10 \mathrm{~min}$ stimulation, the preparations were transferred quickly to freshly made $4 \%$ paraformaldehyde (Sigma, St. Louis, MO) in physiological saline and left at $4^{\circ} \mathrm{C}$ overnight.

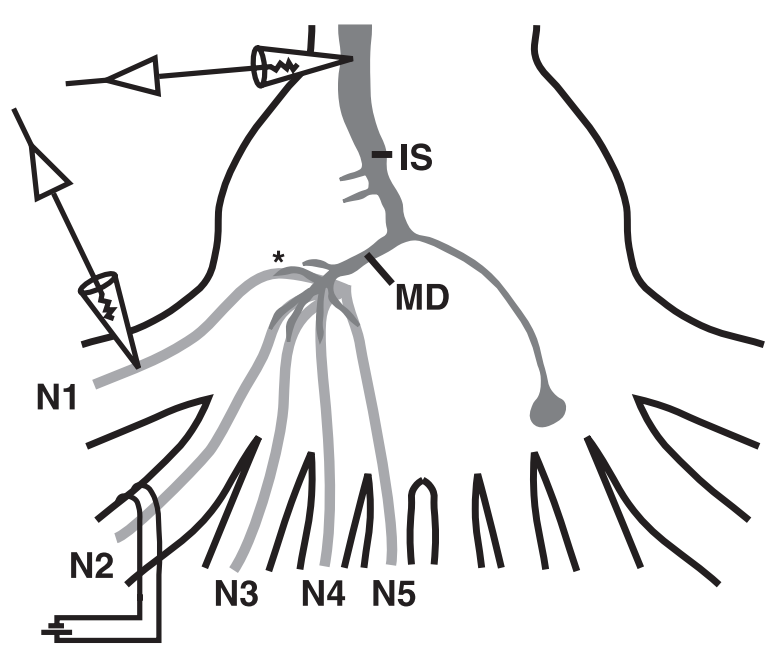

Figure 1. A schematic of the experimental preparation is shown. Stimulating suction electrodes were placed on peripheral nerves as described in Materials and Methods and Results for each experiment (N2 shown). Intracellular recording electrodes in the LG were placed in the initial segment (IS), major dendrite (MD), or in the dendrites near afferent contact points (asterisk). Intracellular recordings from afferents were made near the base of the nerve root where it enters the ganglion (arrows) or near the afferent- $L G$ contacts in the neuropile (asterisk).

To determine the effect of a sustained change in LG membrane potential on dye coupling, the LG was first injected with tracers as above and then voltage clamped at the initial segment with two electrodes to a voltage between -15 and $+40 \mathrm{mV}$ from rest for $10 \mathrm{~min}$. The preparations were then fixed as above.

To determine the effects of activity in a single sensory afferent on dye coupling between the LG and inactive afferents, the LG was filled with tracers as above, and an afferent was penetrated with a $30 \mathrm{M} \Omega$ electrode filled with 0.5\% $3000 \mathrm{MW}$ dextran-linked Cascade Blue anionic (Molecular Probes) in $3 \mathrm{M} \mathrm{KCl}$ (Fig. 1). The afferents were filled by injecting 5-10 $\mathrm{nA}$ of hyperpolarizing DC for $10 \mathrm{~min}$ using an Axoclamp 2B (Axon Instruments, Foster City, CA). Injected afferents were then stimulated using depolarizing current pulses of $5 \mathrm{~ms}$ duration at $5 \mathrm{~Hz}$, at an amplitude $10 \%$ over threshold. Almost every afferent tested would fire at this frequency over the $10 \mathrm{~min}$ period; any that would not were not analyzed. After this, preparations were fixed as above.

Processing and imaging. After fixation, the posterior nerve cords with intact A6 peripheral nerves were removed from the preparation and placed in three $1 \mathrm{~h}$ washes of $0.1 \%$ Triton X-100 (Sigma) in $0.1 \mathrm{M}$ phosphate buffer, $\mathrm{pH} 7.4$ (PBTX). To visualize the NB, the tissues were then placed in a 1:50 solution of streptavidin-linked Alexa Fluor 488 (Molecular Probes) in PBTX and left on a shaker at $4^{\circ} \mathrm{C}$ for $24-36 \mathrm{~h}$. Next, tissues were then washed six times for $1 \mathrm{~h}$ in PBTX and once in distilled water for $15 \mathrm{~min}$, followed by slow dehydration through an ethanol series with $10 \%$ steps to $100 \%$. The nerve cords were then trimmed, placed carefully dorsal side up in well slides, and cleared and mounted in methyl salicylate (Sigma).

Filled nerve cords were imaged on a Zeiss (Thornwood, NY) LSM 510 confocal microscope using $20 \times$ Fluor air interface and $63 \times$ $\mathrm{C}$-Apochromat water interface lenses. For all preparations, full images of all labeled structures were acquired using the $20 \times$ objective and interslice intervals between 4 and $4.1 \mu \mathrm{m}$. Close-ups of regions to be used for analysis were imaged with the $63 \times$ objective at intervals of $0.5 \mu \mathrm{m}$. All images were captured using full resolution $(2048 \times 2048$ pixels $)$ and stored with false colors based on the emission of the fluorophores. Because the characteristics of the microscope varied with environmental conditions, time in use, and other factors, beads of standardized fluorescence (Molecular Probes) were imaged in series with each sample. Tests using these beads (data not shown) revealed that they could be used to correct for fluctuations in microscope performance to obtain reliable intensity readings from this microscope. In most preparations, the dyefilled and dye-coupled neurons were far brighter than any autofluorescence in the tissues, and any preparations in which autofluorescence 
could not be clearly and unambiguously distinguished from filled neurons on the captured images were not used for analysis. Montages of confocal stacks for figures were reconstructed using Adobe Photoshop 7 software (Adobe Systems, San Jose, CA) using TIFF images exported from the Zeiss software. For the figures reproduced in this study, brightness of the images was adjusted if necessary to allow easy visualization of relevant structures after printing.

A measure of dye coupling between the LG and primary afferents was obtained from the ratio of the fluorescence intensity of the primary afferent axon (measured adjacent to its apparent contact point with the LG) to the intensity of the adjacent LG dendrite. Background intensity readings from immediately adjacent areas not containing filled processes were subtracted from each intensity reading before taking the ratio. For the peripheral nerve stimulation and current injection into LG experiments, the primary afferents in each of N1 through N5 were binned and treated as single populations. Trials using one randomly chosen preparation from each stimulus regimen showed that the intensity of individual neurons within nerves was extremely consistent, and therefore only one randomly chosen afferent from each population was measured for analysis. For the single-afferent stimulation experiments, any afferent that could be determined by visual analysis to be brighter than the population of afferents as a whole was measured individually. For each analyzed afferent, those slices containing that axon and adjacent LG dendrites were extracted, and the single slices containing the widest point of each structure were chosen for measurement. Only a single slice was analyzed because we determined that the brightness of NB-linked fluorescence (NBF) was homogenous throughout structures, and we wanted to ensure that the measurements were not skewed by measuring a greater thickness of some structures relative to others. The slice thickness used was much less than the diameter of the smallest afferent axons or LG dendrites analyzed, which gives us confidence that a single slice in the middle of a structure contained a uniform thickness of intracellular space throughout the structure. Intensity readings were gathered as histograms from these slices using the software supplied with the LSM 510 confocal microscope and the public domain NIH Image software (developed at the National Institutes of Health and available at http://rsb.info.nih.gov/ nih-image/) on a Macintosh computer. These histograms were standardized using the data from the calibration beads, and mean intensity ratios between the primary afferent and LG were calculated.

Physiology. Lateral excitatory network physiology was performed by placing one suction electrode on the nerve cord rostral to the terminal ganglion to stimulate the LG and for identifying sensory afferents by the primary afferent depolarization that results from an LG spike (Bryan and Krasne, 1977). One or two suction electrodes were placed on peripheral nerves of A6 to evoke afferent volleys (Fig. 1). Intracellular microelectrodes for recording and current injection had resistances of 15-35 M $\Omega$. Microelectrode filling solutions were $3 \mathrm{~m} \mathrm{KCl}$ for the LG and $2 \mathrm{M} \mathrm{KAc} \mathrm{for}$ afferents. LGs were impaled with one or two microelectrodes in the initial segment in close proximity to A6 and identified by their response to sensory nerve stimulation. Afferent recording microelectrodes were placed in different sensory nerves close to their roots (Fig. 1). Primary afferents were identified by their response to nerve cord stimulation and by their morphology as revealed by injection with NB or Lucifer yellow (LY), followed by fixation and visualization.

To study depolarizing current flow through the LG and back into the afferents, we placed a stimulating suction electrode on N2, then poked either LG dendrites or primary afferents with $60 \mathrm{M} \Omega$ electrodes containing $3 \mathrm{M} \mathrm{KCl}$ and $3000 \mathrm{MW}$ dextran-linked Cascade Blue (Molecular Probes) (Fig. 1). The LG was filled with either TR or TR and NB, and electrode locations were confirmed by iontophoretically injecting 3000 MW dextran-linked Cascade Blue into the cells, followed by quick fixation and visualization as described in Materials and Methods.

Data analysis. Data were analyzed using SigmaStat software (SPSS, Chicago, IL). Changes in afferent populations as a result of the stimulation regimen were analyzed between animals using Kruskal-Wallis oneway ANOVA on ranks and the Mann-Whitney rank-sum test. Differences between afferent populations within animals were analyzed using Friedman repeated-measures ANOVA on ranks and the Wilcoxon signed rank test. The $t$ tests were used to test the effect of $50 \mathrm{~Hz}$ stimula-

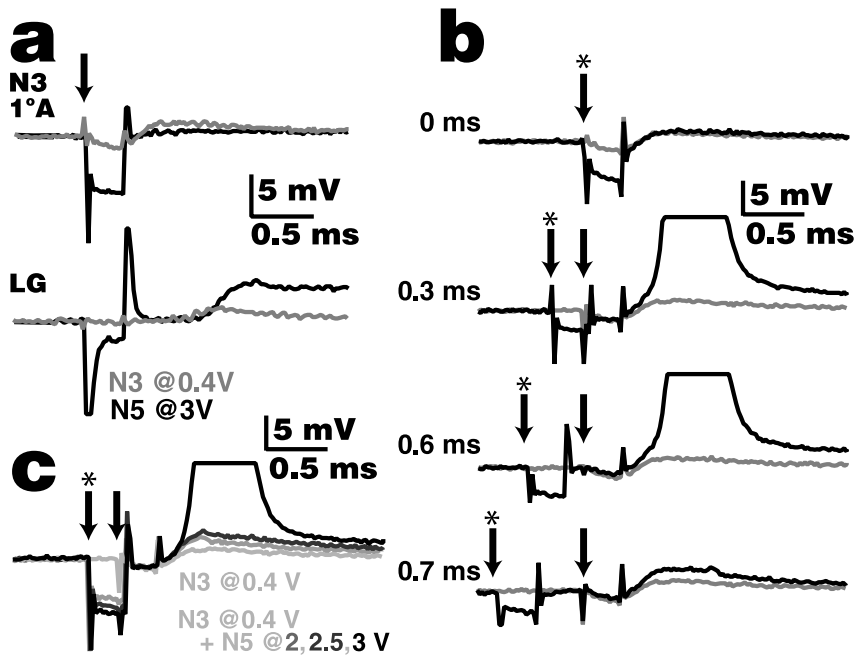

Figure 2. Stimulation of one nerve (N5 in this example) enhances lateral excitation in a distant nerve (N3). $\boldsymbol{a}$, Responses of an N3 afferent and LG to 0.3 ms shocks of N3 ( 0.4 V; gray traces) and to shocks of N5 (3 V; black traces). N3 shock elicits an $\sim 2 \mathrm{mV}$ potential in the afferent axon, recorded in the nerve root, whereas $\mathrm{N} 5$ shock elicits a very small $(<0.5 \mathrm{mV})$ potential. Both shocks elicit an EPSP in the LG at the initial segment, although the N5 shock, being larger, elicits the larger response. The arrow indicates the onset of the nerve shocks. $\boldsymbol{b}$, Lateral excitation is enhanced if N5 shock precedes N3 shock within a discrete, short-latency window. The gray traces are the $\mathrm{N} 3(0.4 \mathrm{~V})$ shocks alone, black traces are the N3 plus N5 (3 V) shocks together at different latencies, the arrows indicate the onset of the N3 shock, and the arrows with asterisks indicate the onset of the N5 shock (latency between shocks is given on each trace). There was no enhancement of the potential if the two shocks were delivered simultaneously (top trace). In this example, the $\mathrm{N} 3$ afferent was recruited only if the $\mathrm{N} 5$ stimulus preceded the $\mathrm{N} 3$ stimulus by $0.3-0.6 \mathrm{~ms}$ (middle two traces). Outside this range, some enhancement of the response recorded in the $\mathrm{N} 3$ afferent occurred between 0.2 and $0.8 \mathrm{~ms}$ latency (e.g., bottom trace, $0.7 \mathrm{~ms}$ ). c, Enhancement of lateral excitation depends on the strength of the previous stimulus. With the N3 (0.4 V) shock (arrow) following the N5 shock (arrow with an asterisk) with the optimal 0.3 ms latency, it required a strong ( 3 V) N5 shock to recruit the afferent (black trace). Lower-voltage shocks to N5 enhanced the potential recorded in the afferent (dark gray traces) over the response from N3 shock alone (light gray trace, lowest response) but did not result in recruitment. All experiments were from the same preparation and cells.

tion on the number of afferents coupled to LG and to test the effect of voltage clamping the $\mathrm{LG}$ at rest on the number of coupled afferents.

\section{Results}

\section{Afferent recruitment between sensory nerves}

Stimulation of a sensory nerve can enhance lateral excitation in a second, distant nerve if the stimulus is of sufficient strength and if the latency between it and a stimulus to the second nerve falls within a discrete window (Fig. 2). In the example shown, shock of $\mathrm{N} 3$ elicited a synaptic potential in an $\mathrm{N} 3$ afferent, recorded in the nerve root, and an LG EPSP, recorded at the initial segment (Figs. $1,2 a$ ). Shock to N5 elicited a smaller N3 afferent potential but a larger LG EPSP. Because there are few direct connections between afferents in different nerves, we believe the potential in the afferent was caused by the LG EPSP-driving retrograde flow of current through the electrical junctions connecting afferents to the LG, forming an antidromic synaptic potential (ASP). The connections between the afferents and LG are believed to be rectifying but normally conducting at rest. The potential elicited in the afferent by $\mathrm{N} 3$ stimulation would therefore be a sum of EPSPs produced by direct connections with other afferents and the ASP produced via the response of the LG to the nerve shock.

Recruitment of an afferent depends on the latency between shocks in a distant nerve and in the nerve containing the afferent. If the N3 and N5 shocks were applied simultaneously, there is no discernable change in the potential recorded in the N3 afferent (Fig. $2 b$, top trace). However, if the latency from the N5 stimulus 


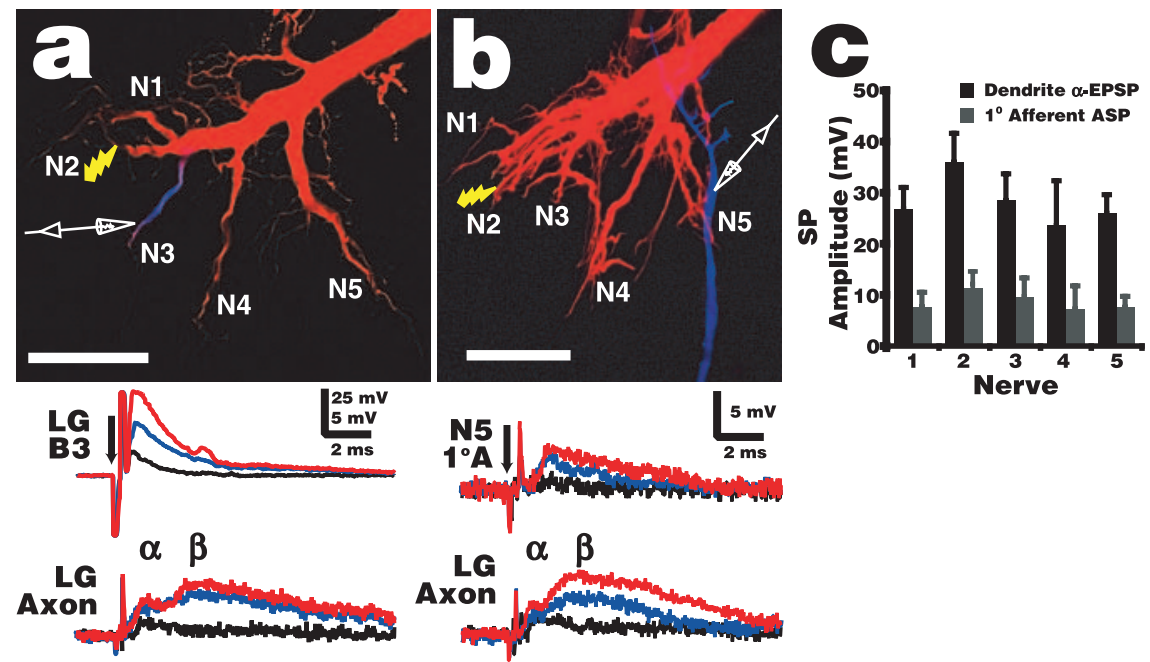

Figure 3. Electrotonic spread of LG EPSPs into neighboring dendrites and antidromically into their presynaptic afferents. $\boldsymbol{a}$, In this example, N2 was shocked, whereas intracellular recording electrodes were placed in the LG initial segment and the LG dendrite branch contacting N3 afferents. The micrograph illustrates the relative locations of the stimulus and the dendrite recording electrode, whereas the traces demonstrate that a very large EPSP was recorded in the N3 branch (LG B3) in response to subthreshold $\mathrm{N} 2$ shocks at $1 \mathrm{~V}$ (black), $1.3 \mathrm{~V}$ (blue), and $1.5 \mathrm{~V}$ (red; $70 \%$ of the LG threshold). The electrode in the initial segment (LG Axon) recorded the typical, much-lower-amplitude biphasic LG EPSPs ( $\alpha$ and $\beta$ component labeled), demonstrating that although high-amplitude EPSPs are maintained in the dendrites, there is substantial decay in the $\alpha$ component before they reach the initial segment. $\boldsymbol{b}$, Similar setup as in $\boldsymbol{a}$, except that the second recording electrode was placed in an N5 primary afferent rather than the N3 LG branch (micrograph). Small shocks to N2 (0.8 V in this example, black trace) that elicit a small EPSP at the LG initial segment do not elicit a detectable ASP in afferents in other nerves. Larger shocks (blue, $1.0 \mathrm{~V}$; red, $1.5 \mathrm{~V}, 70 \%$ of the $L G$ threshold) do elicit afferent ASPs, and these reflect the time course of the LG dendrite EPSPs fairly closely with a short delay. In $\boldsymbol{a}$ and $\boldsymbol{b}$, the LG was filled with TR and NB (data not shown for clarity), and the recording site was identified by injecting Cascade Blue. The arrows indicate stimulus onset. Scale bars, $100 \mu \mathrm{m}$. c, Average synaptic potential (SP) amplitude, with SD, of primary afferent ASPs from each sensory nerve and EPSPs from the corresponding LG dendrites, evoked by N2 shock at $70 \%$ of the LG stimulus threshold ( $n=6$ for each). Afferent ASPs in N2 were not distinguishable from EPSPs elicited from direct afferent-afferent connections; therefore, the amplitudes given here represent the sum result of these two pathways.

to the N3 stimulus was between 0.26 and $0.65 \mathrm{~ms}$, the N3 afferent was recruited to fire (Fig. $2 b$, middle two traces). The $0.39 \mathrm{~ms}$ duration of the recruitment window seen in this example was typical; the range seen in all preparations was from 0.16 to 0.59 $\mathrm{ms}$ with mean $\pm \mathrm{SD}$ of $0.31 \pm 0.21 \mathrm{~ms}(n=7)$. Outside of the recruitment window, we also saw subthreshold enhancement of the potential recorded in the afferent; in this example, enhancement occurred between latencies of 0.2 and $0.8 \mathrm{~ms}$ (Fig. $2 b$, bottom trace).

Recruitment of an afferent also depends on the strength of the stimulus applied to the distant nerve. In the example in Figure $2 c$, a latency of $0.3 \mathrm{~ms}$ between the $\mathrm{N} 5$ and $\mathrm{N} 3$ shocks recruited the afferent if $3 \mathrm{~V}$ was applied to N5. With 2 or $2.5 \mathrm{~V} \mathrm{~N} 5$ shocks, the afferent was no longer recruited, although the potential in the N3 afferent was enhanced, suggesting that these smaller N5 shocks still had a subthreshold effect on the N3 lateral excitatory network. Larger nerve shocks elicit larger EPSPs in the LG; it follows that they would then have a larger effect on afferent ASPs in distant nerves.

The lateral excitatory network formed by direct afferent-afferent connections is crucial for afferent recruitment. Few connections exist between afferents in different nerves (Herberholz et al., 2002; Antonsen and Edwards, 2003), and we never saw recruitment of afferents resulting solely from stimulation of a distant nerve, or from current injection into the LG.

\section{Afferent recruitment is limited to the ipsilateral side}

Because both LG neurons are electrically coupled in the terminal ganglion (Watanabe and Grundfest, 1961; Antonsen and Edwards, 2003), we investigated the possibility that afferent recruit- ment was possible not only between nerves on the same side of the ganglion but across the ganglion to the contralateral LG as well. Recruitment of an ipsilateral afferent was not affected by current injections into the contralateral LG or by subthreshold stimulation of one or more contralateral sensory nerves (data not shown). Sites of bilateral coupling between LGs are physically distant from sites of afferent-LG contact (Antonsen and Edwards, 2003), and the bilateral contacts appear to be too weak to enable cross-ganglionic recruitment of primary afferents.

\section{Dendritic LG EPSPs spread back into unstimulated afferents}

To determine how synaptic potentials resulting from stimulation of one nerve spread through the dendritic tree of the LG and into the afferents that synapse on it, we recorded from the initial segment of the LG axon, distal LG dendrites, and primary afferent axons near their LG contact points (Fig. 1). After making the recordings, the LG was filled with 10,000 MW dextranlinked TR and NB by picospritzing from the electrode in the initial segment. Recording sites on LG dendrites or primary afferents were identified, based on our knowledge of circuit anatomy (Antonsen and Edwards, 2003), by iontophoretically injecting 3000 MW dextran-linked Cascade Blue; afferents were filled completely (5-10 min), but LG dendrites were filled with a $30 \mathrm{~s}$ pulse only, then fixed quickly to prevent diffusion through the cell (Fig. $3 a, b)$.

N2 shock elicited large, short-latency EPSPs in LG dendrites that quickly decayed (Fig. $3 a$ ). The large, long-lasting $\beta$ component that dominates recordings made at the initial segment is hard to distinguish in the dendrites, possibly because it originates mostly from cells that contact the LG close to the initial segment (Antonsen and Edwards, 2003), and it therefore is masked by the very large $\alpha$ component in the dendrites. Primary afferent ASPs recorded near LG contact points were up to $15 \mathrm{mV}$ in size in some cells and reflected the shape and time course of the dendrite EPSPs fairly closely, with a short delay (Fig. 3b). This delay was likely attributable in part to conduction through the gap junctions connecting the cells and partly to the distance between the LG dendrite recording site and the afferent contact points. The onset of ASPs recorded from different preparations was between 0.12 and $0.47 \mathrm{~ms}$ after the shock was delivered $(0.26 \pm 0.04 ; n=$ $31)$, whereas the peak was reached after $0.36 \pm 0.09 \mathrm{~ms}$. This is very close to the beginning of the interstimulus time window for enhancement of lateral excitation in distant nerves $(0.37 \pm 0.11$ $\mathrm{ms} ; n=7$ ) (Fig. 2). It stands to reason that enhancement of lateral excitation should occur when the afferent is depolarized above some threshold. Therefore, the peak of the ASP should occur near the beginning of the window, because the rise of the ASP is quite fast, and the slow decay of the ASPs (Fig. 3b) will allow for the time window over which enhancement was observed to occur. These points support the idea that the increase in lateral excitation is attributable to ASPs passing to distant afferents from the 


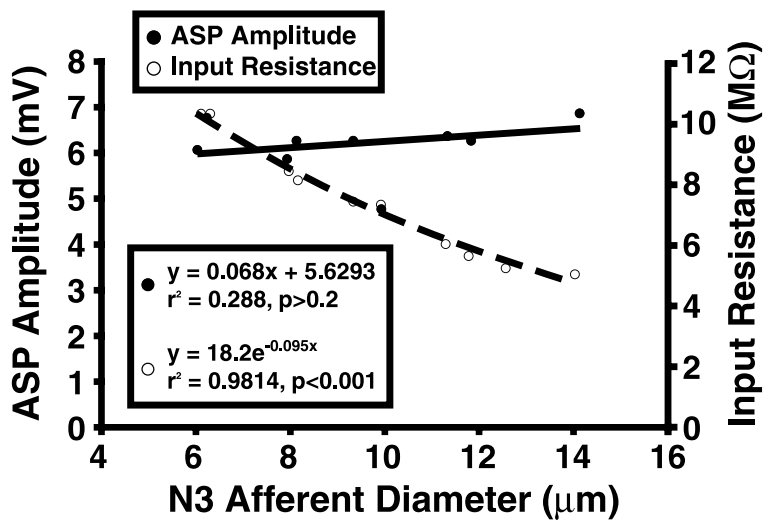

Figure 4. Antidromic synaptic potential amplitude in N3 primary afferents in response to N2 shocks is not dependent on afferent size, measured as axon diameter. Input resistances and ASP sizes were measured from 10 afferents, ranging in size from 6 to $14 \mu \mathrm{m}$. Although input resistance decreased with larger size as expected, ASP amplitude did not change significantly.

LG. Both LG EPSPs and afferent ASPs increase in size with increasing stimulus strength, suggesting that EPSP size (driving force) contributes to the size of the ASP recorded in the afferent.

The LG EPSP elicited by an N2 shock attenuated only slightly, and not significantly, with distance from the stimulus site (Fig. $3 c$ ). This is not surprising, because the main LG dendrites that form the contact points for afferents from each nerve are all very large and branch off of a common large dendritic trunk, so physical and electrical distances are not substantially different between them. However, in the fine distal branches of the N2 dendrite itself, EPSPs reach $80 \mathrm{mV}$ in response to subthreshold nerve shocks (B. L. Antonsen and D. H. Edwards, unpublished results). Similarly, ASP amplitudes in afferents decayed slightly, but not significantly, with distance from the stimulus site. The potentials recorded from $\mathrm{N} 2$ afferents were a combination of ASPs and EPSPs elicited via direct afferent-afferent connections and so were not surprisingly larger than from any other nerve.

\section{Antidromic transmission is independent of afferent size}

Primary afferents that are dye coupled to the LG have axon diameters from 1 to $14 \mu \mathrm{m}$ (Antonsen and Edwards, 2003). To determine whether this size difference might account for the variation in ASP amplitudes recorded in response to sensory nerve stimulation, ASPs produced by antidromic LG spikes were recorded in N3 afferents ranging from 6 to $14 \mu \mathrm{m}$ (Fig. 4). Antidromic LG spikes, elicited by current injection into the LG, were used to elicit the ASPs to provide a consistent, large stimulus. All recordings were made in the neuropil very near the LG contact point (Fig. 1). ASPs produced under these circumstances are $\sim 6-7 \mathrm{mV}(6.29 \pm 0.64 ; n=10)$, smaller than the maximum of $12-15 \mathrm{mV}(13.14 \pm 0.86 ; n=14)$ seen as a result of strong nerve stimulation in N3 afferents, and typically last $<2 \mathrm{~ms}$. Although the measured afferent input resistance dropped by $\sim 50 \%$ over this range of increasing axon diameter, the ASP amplitude remained constant. This would occur if the coupling resistance between the afferent and LG were a constant fraction of the input resistance of the afferent over the range of afferent sizes.

\section{Dye coupling reveals interactions between afferents through LG dendrites}

Dye coupling between the LG and other elements of the escape circuit occurs when the LG is filled with NB (Fig. 4) (Herberholz et al., 2002; Antonsen and Edwards, 2003). Approximately 250
$(255 \pm 16.3 ; n=6)$ afferents from N1 to N5 in A6 were found with detectable levels of NBF from dye coupling with the LG. Not all primary afferents dye couple with the LG; backfilling a sensory nerve with a fluorescent tracer revealed that $\sim 50-70 \%$ of the primary afferents in any nerve dye couple with LG (Antonsen and Edwards, unpublished results). Motor neurons (the brightly filled somata in Fig. 4A), premotor neurons, interneurons, and the local LG neuron all dye coupled very strongly with the projecting LG in unstimulated preparations (Antonsen and Edwards, 2003). However, we found that NB (positively charged) dye coupling from the LG to the afferents, measured as relative afferent NBF, increased as a result of LG depolarization. We used this to design a series of experiments aimed at revealing current flow through the entire LG and back into afferents based on patterns of dye coupling.

In unstimulated preparations, all measured afferents had similar NBF intensity, independent of location (Friedman test; $p>$ $0.1 ; n=6)$. In three preparations in which we measured the fluorescent intensity of all dye-coupled afferents relative to the LG, the range of intensities was not $>15 \%$ of the mean (Fig. $5 b$ ). Afferents contacting the LG range between 1 and $14 \mu \mathrm{m}$ axon diameter, which suggests that larger afferents must receive more NB from the LG to reach the same internal concentration and therefore the same intensity of NBF.

Electrical shocks applied to $\mathrm{N} 2$ at $70 \%$ of the threshold voltage of the LG induced significant changes in relative afferent NBF in each peripheral nerve (Fig. $5 c, d$ ) (Kruskal-Wallis test; $p<0.001$ for each nerve; $n=6$ ). Relative afferent NBF increased with stimulus frequency, the most rapid increase occurring between 0 and $10 \mathrm{~Hz}$ and 10 and $20 \mathrm{~Hz}$ (Mann-Whitney test; $p<0.01$ for N1, $\mathrm{N} 2, \mathrm{~N} 3$, and N5; $p<0.02$ for N4). Above $20 \mathrm{~Hz}, \mathrm{NBF}$ intensity continued to increase, but more slowly, with significant differences only between 20 and $100 \mathrm{~Hz}$ ( $p<0.025$ in all nerves). We believe this increase in dye coupling results from the depolarizing EPSPs in the LG dendrites driving the positively charged NB into primary afferents as it produces ASPs in those afferents. To test this, we injected the LG with negatively charged LY instead of NB and saw strong dye coupling in three unstimulated preparations and no detectable increase as a result of the $20 \mathrm{~Hz}$ stimulation of $\mathrm{N} 2$ in three other preparations (data not shown). Because the junctions connecting afferents with the LG are rectifying, and therefore change properties in response to changes in transsynaptic voltage, it is still possible that some conformational change occurs in the junctions that will change their permeability to small molecules. However, we believe the data presented here and later in Results suggest that the iontophoretic explanation is the more likely one.

As with the other nerves, fluorescent intensity in N4 increased with $\mathrm{N} 2$ stimulus frequency, but the magnitude of the increase was only $\sim 10 \%$ of that seen in the other nerves (Fig. $5 d-f$ ). When $\mathrm{N} 4$ was stimulated, dye coupling of N4 afferents to the LG was still less strong than that of afferents in other nerves, but the difference between them was much less (Table 1). These results may be attributable to the necessity in these preparations of cutting the peripheral N4 branches fairly close to the ganglion to expose the nerve roots for the stimulating suction electrode, but trials leaving the $\mathrm{N} 4$ innervation intact did not conclusively prove this (data not shown). Although N1 branches were also cut during the dissection, the length of root between the ganglion and cut was approximately three to four times that of N4.

Similar to the results seen when recording from single afferents (Fig. 3c), N2 stimulation resulted in small, but in this case significant, differences in relative NBF intensity between nerves 

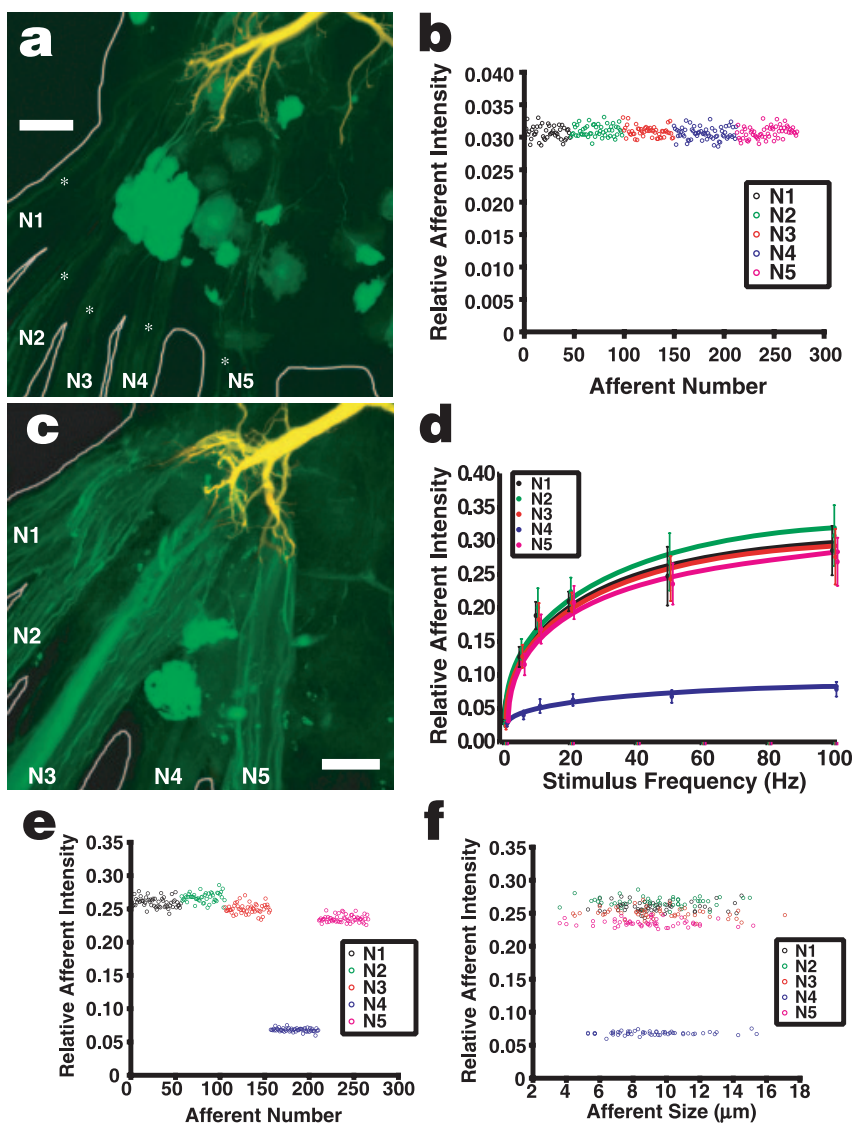

$f$
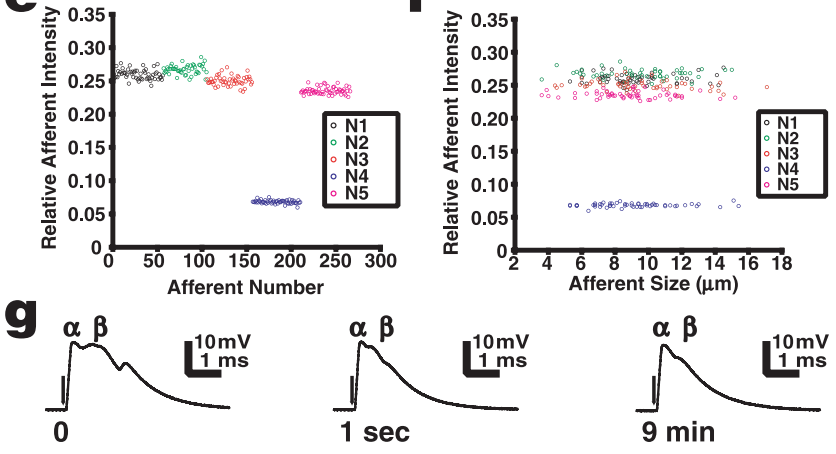

Figure 5. Sensory nerve shock increases NB dye coupling from the $L G$ to primary afferents in A6. The LG was injected with a pair of tracers, TR (red) and NB (green), which combined to make the LG appearyellow; cells that dye couple to the LG appear green. $\boldsymbol{a}$, A preparation in which the LG was filled with the tracers but no additional stimulus was applied. The LG dendrites can be seen at the top of the micrograph, and the somata of dye-coupled motor neurons appear bright green [see Antonsen and Edwards (2003) for complete details of the anatomy]. The axons of primary afferents that dye coupled to the $L G$ can be seen entering the ganglion from each peripheral nerve (asterisks), but they are quite faint in unstimulated preparations. $\boldsymbol{b}$, Within a single preparation, NBF of afferents relative to $L G$ was highly consistent independent of the location of the afferent (nerve). Afferents are plotted in the order they were measured and are color-coded to their nerve. See Materials and Methods for details on calculating relative afferent fluorescent intensity. c, Afferents appear much brighter in preparations that have been stimulated with $\mathrm{N} 2$ shocks; in this example, $20 \mathrm{~Hz}$ stimulation was applied for 10 min at an amplitude of $70 \%$ of the $L G$ threshold voltage. $d$, Dye coupling between afferents in all nerves and the LG increased significantly with the frequency of N2 stimulation (Kruskal-Wallis test; $p<0.001$ for each nerve). Points show means \pm SD $(n=6)$. e, Relative afferent NBF intensity remained consistent among afferents of any given nerve after stimulation. This example is from a single preparation stimulated at $50 \mathrm{~Hz}$. $f$, In the same preparation, relative afferent NBF intensity did not depend on afferent size (axon diameter). $\boldsymbol{g}$, Intracellular recordings from the major dendrite of the LG near the initial segment show that the $\alpha$ component of the LG EPSP evoked by $20 \mathrm{~Hz}$ $\mathrm{N} 2$ stimulation remained constant over the stimulus period (times given are from start of stimulation), whereas the later $\beta$ EPSP components quickly depress. This pattern was similar for each stimulus frequency. The arrows indicate the beginning of the stimulus, although the artifacts are too small to see on these traces. Scale bars, $50 \mu \mathrm{m}$.

(Friedman tests; $p<0.01$ for each stimulus frequency). Relative NBF intensity was strongest in afferents of the stimulated nerve and decreased somewhat with distance (Fig. 5e). N2 afferents had the strongest dye coupling with LG by a small but somewhat significant amount at 5,20, and $50 \mathrm{~Hz}$ stimulation (Wilcoxon test; $p<0.04$ for all cases), whereas at 10 and $100 \mathrm{~Hz}, \mathrm{~N} 2$ afferents had the strongest dye coupling, but not significantly so (Fig. $5 d$ ). Afferent NBF in N1 and N3 was very similar and slightly lower than in N2, and N5 afferents were slightly weaker than N1 or N3 afferents, but this was significant only at $5 \mathrm{~Hz}$ (Wilcoxon test; $p<$ 0.04). N4 afferents had substantially lower dye-coupling strength with the LG than any other afferents at any stimulus regimen other than rest (Wilcoxon test; $p<0.04$ for all cases). We believe that dye coupling decreases with distance from the stimulus site for the same reasons outlined for ASP amplitude above: slight decay of the LG EPSP as it passes through LG dendrites.

As in the unstimulated preparations, dye-coupling strength between the LG and individual afferents was highly consistent within nerves (Fig. $5 c, e$ ). Afferent size also had no effect on intensity (Fig. 5f). Nerve shock promoted dye coupling but did not change the numbers of primary afferents coupled to the LG in the terminal ganglion. For example, the number of coupled afferents at $50 \mathrm{~Hz}$ stimulation was $257.1 \pm 12.5$ and at rest $255.5 \pm 16.3(t$ test; $n=6$ for each group; $p>0.85$ ).

Electrophysiological records from the major dendrite of the LG (Fig. 1) reveal that 22-30 $\mathrm{mV}$ EPSPs were elicited by nerve shocks at $70 \%$ of the LG threshold (Fig. $5 g$ ). Discrete EPSPs were recorded even at $100 \mathrm{~Hz}$ stimulation, although at 50 and $100 \mathrm{~Hz}$ the potential of the cell would not quite return to rest between shocks, and we recorded a 3-4 mV sustained depolarization. The short-latency, monosynaptic $\alpha$ EPSP from primary afferents retained its initial amplitude through the stimulus period, whereas the later disynaptic $\beta$ EPSP from mechanosensory interneurons appeared in only the first few shocks. This is in agreement with Krasne (1969), who found that $\beta$ was essentially eliminated by synaptic depression after 10-100 shocks.

\section{LG depolarization mimics the effects of sensory stimulation on dye coupling}

To determine whether depolarization of the LG alone could increase NBF in coupled sensory afferents, we measured the relative change in afferent fluorescence produced by sustained voltageclamp steps applied to the initial segment of the LG filled with NB and 10,000 MW TR. The LG was voltage clamped to membrane potentials ranging from -15 to $+40 \mathrm{mV}$ from rest for $10 \mathrm{~min}$. The resting membrane potential ranged from -92 to $-95 \mathrm{mV}$. Voltage clamping the LG at rest resulted in similar levels of afferent NBF in each nerve (Table 2), and these levels were not different from those seen in any nerve in the unstimulated preparations from the N2 shock experiments, suggesting that voltage clamping alone has no effect ( $t$ test; $p>0.7$ for each nerve). NBF intensities in afferents significantly differed depending on membrane potential (Table 2) (Kruskal-Wallis test; $p<0.001$ for each nerve). Significant afferent NBF increases from rest levels were elicited in each nerve by depolarizations $>5 \mathrm{mV}$ (Mann-Whitney test; $p<0.01$ ). Afferent-relative NBF was similar in each nerve at any given stimulus level, except N4 afferents, which showed an increase, but the amount of relative NBF was significantly less $(\sim 30-50 \%)$ than in the other nerves (Table 2$)$. Hyperpolarization of the LG by $15 \mathrm{mV}$ decreased the relative NBF in each nerve, but these differences were not significant (MannWhitney test; $p>0.1$ for each nerve).

\section{Single afferent stimulation results in localized increases in dye coupling}

Stimulating single N3 afferents with trains of intracellular superthreshold current pulses delivered far out in the nerve root elicited increased dye coupling between the LG and populations of afferents in the immediate vicinity of the stimulated afferent (Fig. 
$6 a)$. Furthermore, the size of the stimulated afferent influenced the extent of the area over which increased dye coupling could be detected. Stimulation of larger afferents resulted in increased dye coupling between the LG and a larger number of nearby afferents and in an increased distance through LG dendrites, over which we could detect the effect (Fig. 6b). The distance was measured through the center of the LG dendrites from the contact point of the stimulated afferent to the contact points of afferents with increased relative NBF. We suggest that stimulation of larger afferents produces greater dye coupling between the LG and neighboring afferents, because the larger afferents evoke larger EPSPs in the LG, as could be predicted from the ASP data covered above. Measuring EPSP size as a result of singleafferent stimulation directly proved problematic because of the difficulty of standardizing electrode placement relative to a single synapse. However, larger EPSPs should drive greater amounts of NB into nearby unstimulated afferents. This, the ASP data, and the whole ganglion dye-coupling data described earlier in Results suggest that contacts are stronger between the LG and larger afferents.

Previous studies found that primary afferents are dye coupled to other afferents within the same nerve through the lateral excitatory network (Herberholz et al., 2002; Antonsen and Edwards, 2003). It is possible that some of the NB in nonstimulated afferents seen in Figure $6 a$ reached there through interafferent coupling from the stimulated afferent rather than directly from the LG. LY injected into the stimulated afferent of two preparations revealed dye coupling between that afferent and some, but not all, nearby afferents that received increased NB dye coupling as a result of single-afferent stimulation. Two of seven afferents directly dye coupled with the stimulated afferent in one preparation, and two of eight in the other; $\mathrm{NB}$ was linked to Cascade Blue in these experiments (data not shown). We conclude that spikes in single afferents can evoke EPSPs in the LG dendrites that are sufficiently large to induce an increase in dye coupling to neighboring presynaptic afferents.

\section{Discussion}

Attack by a predator on the crayfish's tailfan directly excites a population of primary sensory afferents that project centrally into the terminal abdominal ganglion (Edwards et al., 1999; Herberholz et al., 2004). Each half of the tailfan is mapped through five major nerves onto the major branches of the dendritic tree of the LG in a somatotopic manner, so that afferents at the center of the attack converge onto the tips of one or two of major branches (Antonsen and Edwards, 2003). The afferents excite LG through mixed, but primarily electrical, synapses (Zucker, 1972; Araki and Nagayama, 2003) and also excite a population of sensory interneurons through nicotinic cholinergic synapses (Miller et al., 1992). The electrical synapses appear to be rectifying but normally open (i.e., conducting) (Edwards et al., 1991), so that afferent spikes drive depolarizing current directly into the LG to create large EPSPs in the distal dendrites.

The electrical synapses also allow depolarizing current to flow antidromically into the afferent terminals when the LG is depolarized by injected current (Herberholz et al., 2002). This increases the excitability of unstimulated afferents and enhances their recruitment through the lateral excitatory network connecting afferent central dendrites through nonrectifying electrical synapses (Antonsen and Edwards, 2003). It was suggested that the LG EPSP could facilitate that recruitment in the same manner as the injected depolarizing current, by passing antidromically into the afferents (Herberholz et al., 2002).

The results presented here confirm that suggestion and extend it to show that EPSPs produced by synaptic inputs from one nerve can spread and help recruit afferents in other ipsilateral nerves (Fig. 2). In this way, the LG provides a current path that enables excitation to spread between populations of afferents from different nerves, although there are very few direct afferentto-afferent connections between them (Antonsen and Edwards, 2003). LG EPSPs elicited by afferent input spread throughout the distal LG dendrites and feed back through gap junctions to create ASPs in afferents of other lateral excitatory networks, thus extending the reach of lateral excitation. The recruited afferents then add to the excitation of the LG and other mechanosensory interneurons that project rostrally along the ventral nerve cord toward the brain (Herberholz et al., 2002). Before some of these interneurons (e.g., interneurons A, C) project out of the ganglion, they synapse onto the LG at proximal sites that are far from the distal afferent contact sites (Antonsen and Edwards, 2003). These proximal inputs create the delayed $\beta$ wave of depolarization that sums with the $\alpha$ EPSP produced earlier by the afferents to bring LG to threshold (Krasne, 1969).

A similar antidromic excitatory mechanism may enable the M-cell to spread excitation among VIIIth nerve afferents in fish (Pereda et al., 1995). Unstimulated VIIIth nerve afferent terminals that synapse onto the $\mathrm{M}$-cell are depolarized by antidromic currents produced by M-cell EPSPs that were evoked by inputs from other afferents. This ASP is then enhanced by local active responses in the afferent that can bring it to threshold (Curti and Pereda, 2004).

\section{Mechanism: a neuropilar network of electrical synapses and active sites}

The electrical synapses that link the afferent terminals to each other and to the LG dendrites create a network of active sites for a chain reaction mechanism within the neuropile. The mechanism contains several elements: (1) the lateral excitatory network; 

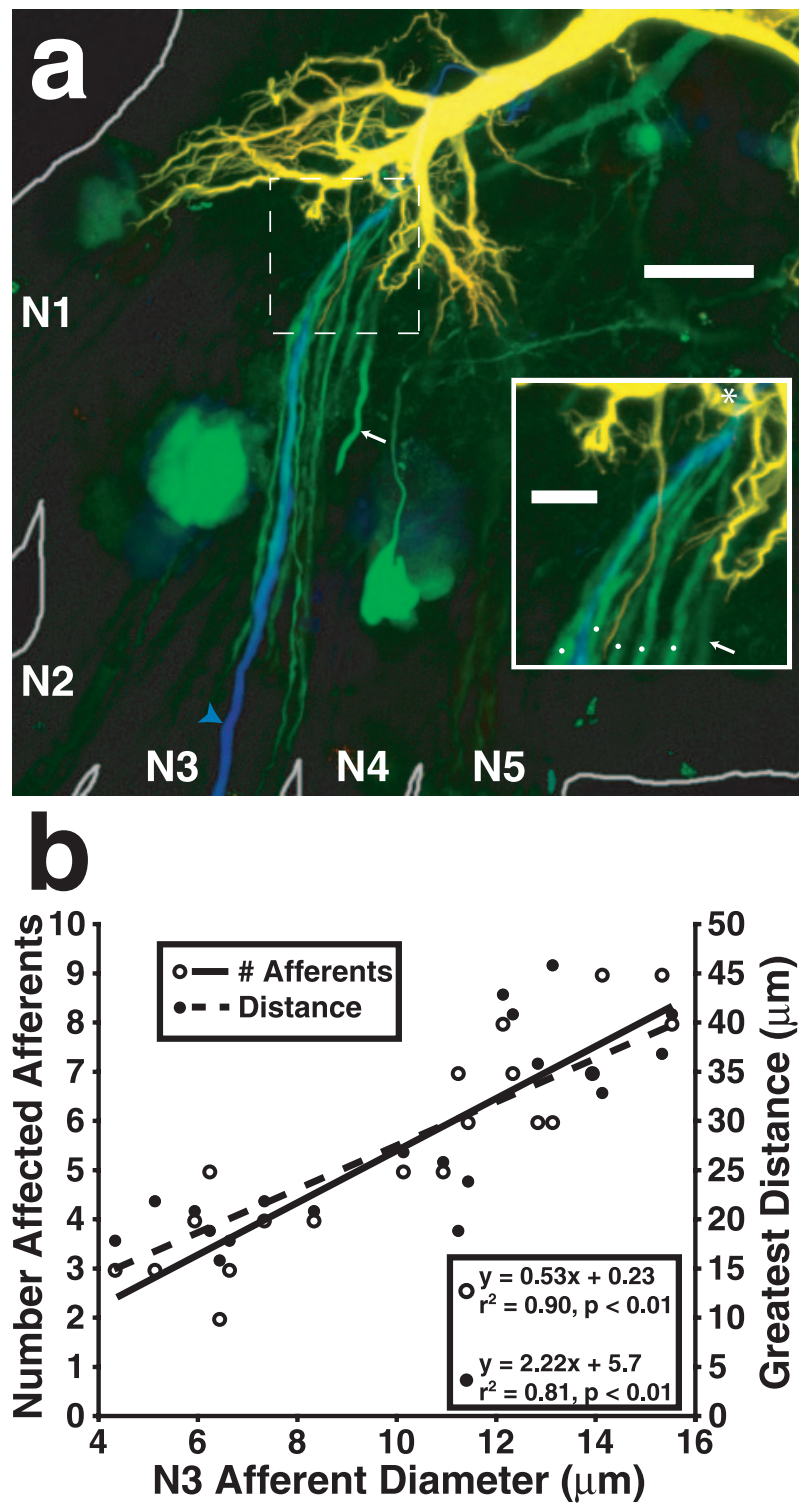

Figure 6. Stimulation of single $\mathrm{N} 3$ afferents at $5 \mathrm{~Hz}$ for 10 min increased $\mathrm{LG}$ dye coupling with neighboring afferents. Both the number of affected neighboring afferents and the distance over which the effect was detected, measured as distance along $L G$ dendrites between the contact points of the stimulated and affected afferents, increased with increasing size of the stimulated afferent. $\boldsymbol{a}$, A confocal photomicrograph of A6 showing the effect of stimulation of a single N3 afferent (filled with 3000 MW Cascade Blue; blue arrowhead) on NB (green) dye coupling from the $L G$ to neighboring afferents. The area outlined by the dashed box is enlarged in the inset. In this case, five neighboring afferents had a detectable increase in relative NBF (white dots in inset). All of the afferents converge onto the $L G$ in close proximity to the stimulated afferent (asterisk in inset). The process indicated by the arrow is a primary neurite of a motor neuron, the cell body of which is not within this stack of slices. The LG was injected with NB and TR. Scale bars: $50 \mu \mathrm{m}$; inset, $10 \mu \mathrm{m} . \boldsymbol{b}$, Graph showing the number of afferents with detectable increases in relative NBF (left ordinate) and the greatest distance from the stimulated afferent along $L G$ dendrites over which the effect could be detected (right ordinate) as functions of the size of the stimulated afferent. Both the number of affected afferents and the distance over which the effect occurs increase with stimulated afferent size.

(2) the passive spread of EPSPs between the LG dendrites; (3) the orthodromic and antidromic conduction of synaptic currents between LG and the afferents; and (4) the excitability of the unstimulated afferents. As we have described previously, the network operates by providing current paths for the LG EPSP and the action currents of stimulated afferents to excite unstimulated afferents and the LG. A chain reaction of afferent excitation can occur when a critical density of afferents are excited within a short period of time. Spikes produced by recruited afferents spread the excitation through the network and add to the EPSP in the LG. They also help recruit mechanosensory interneurons that then provide additional input to the LG that can be decisive in bringing it to threshold.

Although synaptic currents can flow bidirectionally between the LG and the afferents, the network as a whole is polarized by the asymmetry between the afferents and LG. The network operates to select a small set of stimulus conditions to excite the LG. The firing of the LG is the endpoint of the operation of the network; short-latency recurrent inhibition evoked by an LG spike dampens the excitability of the network elements (Roberts, 1968; Kennedy et al., 1974, 1980).

The positive feedback signal amplification that occurs in the network provides an example of the kind of signal processing that can occur within neuropile. The similar results from invertebrates and vertebrates, and the increasing awareness of the importance of gap junctions in the vertebrate CNS, raise the possibility that this mechanism of signal amplification may be common; for example, retrograde chemical transmission has been found recently to play a role in cerebellar long-term depression (Duguid and Smart, 2004), in developing mammalian retina (Syed et al., 2004), and in the rat substantia nigra (Yanovsky et al., 2003). It seems reasonable to expect that other examples using electrical transmission exist and may soon be found.

\section{Function: amplification, latency shortening, and priming}

The network amplifies the inputs of the LG by recruiting unstimulated afferents that enhance the LG EPSP both directly and indirectly through mechanosensory interneurons. The amplification both helps the LG to reach threshold and to reach it sooner. In doing so, it shortens the latency of the response of the LG and thereby that of the escape tailflip. The network also enables initially stimulated areas of the network to prime distant, unstimulated areas to respond to shortly arriving stimuli. Receptors at the center of the predator's strike will respond to it first, and more distant receptors will respond later either as the stimulus reaches them or as the shock spreads across the tailfan through the mechanical coupling of the telson and uropods. The spread of the EPSP away from its site of origin in the LG through the dendritic tree acts to prime afferents in other nerves for recruitment by additional inputs provided by momentarily later stimuli.

The time window for priming lateral excitation by ASPs is both narrow and at short latency from the initial stimulus. This appears to be a result of the shape of the EPSP in LG dendrites; the short-latency, phasic $\alpha$ component of the EPSP is much more prominent than the later, slower $\beta$ component (Fig. 3). Priming occurs coincident with the arrival of the $\alpha$ component at distant LG dendrites.

\section{Effect on spatial resolution}

The lateral excitatory network may contribute to spatial localization of an attack on the abdomen. Although the LG and the tailflip it triggers are both indifferent to the direction or site of attack on the abdomen, information about the stimulus location would guide the highly directional escape swimming movements that follow the LG tailflip. Primary afferents project a spatiotopic map of the tailfan onto LG dendrites (Calabrese, 1976; Antonsen and Edwards, 2003). In previous reports, we described how afferent-to-afferent connections are primarily between afferents within single sensory nerves (i.e., between afferents that innervate 
common areas of the tailfan) (Herberholz et al., 2002; Antonsen and Edwards, 2003). Evidence presented here adds to this by showing that ASPs from the LG are relatively stronger in areas closer to the initial stimulus. This indicates that afferent recruitment produced by the lateral excitatory network and by ASPs from the LG will be relatively stronger in afferents in the portion of the sensory field receiving the strongest stimulus. Stimuli strong enough to excite the LG will excite a broad field of receptors across the tailfan, making identification of the spatial focus of attack difficult. The amplification provided by the network will be enhanced toward the points of strongest stimulus relative to others, and the amplified responses will then be conveyed to the ensemble of mechanosensory interneurons (e.g., interneurons A, $\mathrm{C}$, and others). The pattern of their response may then enable the animal to identify the point of attack (Cuntz et al., 2003).

\section{Lateral excitation and escape}

The short latency of the LG escapes is critical for the animal's survival: when attacked by dragonfly nymphs, crayfish using LGmediated escape tailflips were more successful in avoiding capture than those using non-giant-mediated tailflips that have a slightly longer latency (Herberholz et al., 2004). We conclude that the role of the lateral excitatory network in reducing the latency of an LG-mediated escape is probably more adaptive than preserving spatial resolution for stimuli of this sort.

\section{References}

Antonsen BL, Edwards DH (2003) Differential dye coupling reveals lateral giant escape circuit in crayfish. J Comp Neurol 466:1-13.

Araki M, Nagayama T (2003) Direct chemically mediated synaptic transmission from mechanosensory afferents contributes to habituation of crayfish lateral giant escape reaction. J Comp Physiol [A] 189:731-739.

Bryan JS, Krasne FB (1977) Presynaptic inhibition: the mechanism of protection from habituation of the lateral giant fibre escape response. J Physiol (Lond) 271:369-390.

Calabrese RL (1976) Crayfish mechanoreceptive interneurons. Part 1. The nature of ipsilateral excitatory inputs. J Comp Physiol [A] 105:83-102.

Cuntz H, Haag J, Borst A (2003) Neural image processing by dendritic networks. Proc Natl Acad Sci USA 100:11082-11085.

Curti S, Pereda AE (2004) Voltage-dependent enhancement of electrical coupling by a subthreshold sodium current. J Neurosci 24:3999-4010.

Duguid IC, Smart TG (2004) Retrograde activation of presynaptic NMDA receptors enhances GABA release at cerebellar interneuron-Purkinje cell synapses. Nat Neurosci 5:419-420.

Edwards DH, Heitler WJ, Leise EM, Fricke RA (1991) Postsynaptic modu- lation of rectifying electrical synaptic inputs to the LG escape command neuron in crayfish. J Neurosci 11:2117-2129.

Edwards DH, Heitler WJ, Krasne FB (1999) Fifty years of a command neuron: the neurobiology of escape behavior in the crayfish. Trends Neurosci 22:153-161.

Hatton GI, Yang QZ (1994) Incidence of neuronal coupling in supraoptic nuclei of virgin and lactating rats: estimation by neurobiotin and lucifer yellow. Brain Res 650:63-69.

Herberholz J, Antonsen BL, Edwards DH (2002) A lateral excitatory network in the escape circuit of crayfish. J Neurosci 22:9078-9085.

Herberholz J, Sen MM, Edwards DH (2004) Escape behavior and escape circuit activation in juvenile crayfish during prey-predator interactions. J Exp Biol 207:1855-1863.

Johnson DA, Mills SL, Haberecht MF, Massey SC (2000) Dye coupling in horizontal cells of developing rabbit retina. Vis Neurosci 17:255-262.

Kennedy D, Calabrese RL, Wine JJ (1974) Presynaptic inhibition: primary afferent depolarization in crayfish neurons. Science 186:451-454.

Kennedy D, McVittie J, Calabrese R, Fricke RA, Craelius W, Chiapella P (1980) Inhibition of mechanosensory interneurons in the crayfish. I. Presynaptic inhibition from giant fibers. J Neurophysiol 43:1495-1509.

Krasne FB (1969) Excitation and habituation of the crayfish escape reflex: the depolarizing response in lateral giant fibres of the isolated abdomen. Exp Biol 50:29-46.

Laughlin SB (2002) Retinal function: coupling cones clarifies vision. Curr Biol 12:833-834.

Miller MW, Vu ET, Krasne FB (1992) Cholinergic transmission at the first synapse of the circuit mediating the crayfish lateral giant escape reaction. J Neurophysiol 68:2174-2184.

Peinado A, Yuste R, Katz LC (1993) Extensive dye coupling between rat neocortical neurons during the period of circuit formation. Neuron 10:103-114.

Pereda AE, Bell TD, Faber DS (1995) Retrograde synaptic communication via gap junctions coupling auditory afferents to the Mauthner cell. J Neurosci 15:5943-5955.

Roberts A (1968) Recurrent inhibition in the giant-fibre system of the crayfish and its effect on the excitability of the escape response. J Exp Biol 48:545-567.

Syed MM, Lee S, He S, Zhou ZJ (2004) Spontaneous waves in the ventricular zone of developing mammalian retina. J Neurophysiol 5:1999-2009.

Watanabe A, Grundfest H (1961) Impulse propagation at the septal and commissural junctions of crayfish lateral giant axons. J Gen Physiol 45:267-308.

Yanovsky Y, Mades S, Misgeld U (2003) Retrograde signaling changes the venue of postsynaptic inhibition in rat substantia nigra. Neuroscience 2:317-328.

Zucker RS (1972) Crayfish escape behavior and central synapses. I. Neural circuit exciting lateral giant fiber. J Neurophysiol 35:599-620. 